\title{
Article \\ Between the Classes of Soft Open Sets and Soft Omega Open Sets
}

\section{Samer Al Ghour}

\section{check for}

updates

Citation: Al Ghour, S. Between the Classes of Soft Open Sets and Soft

Omega Open Sets. Mathematics 2022, 10, 719. https://doi.org/10.3390/

math10050719

Academic Editor: Apostolos Syropoulos

Received: 16 January 2022

Accepted: 21 February 2022

Published: 24 February 2022

Publisher's Note: MDPI stays neutral with regard to jurisdictional claims in published maps and institutional affiliations.

Copyright: (C) 2022 by the author. Licensee MDPI, Basel, Switzerland. This article is an open access article distributed under the terms and conditions of the Creative Commons Attribution (CC BY) license (https:// creativecommons.org/licenses/by/ $4.0 /)$.
Department of Mathematics and Statistics, Jordan University of Science and Technology, Irbid 22110, Jordan; algore@just.edu.jo

\begin{abstract}
In this paper, we define the class of soft $\omega^{0}$-open sets. We show that this class forms a soft topology that is strictly between the classes of soft open sets and soft $\omega$-open sets, and we provide some sufficient conditions for the equality of the three classes. In addition, we show that soft closed soft $\omega$-open sets are soft $\omega^{0}$-open sets in soft Lindelof soft topological spaces. Moreover, we study the correspondence between soft $\omega^{0}$-open sets in soft topological spaces and $\omega^{0}$-open sets in topological spaces. Furthermore, we investigate the relationships between the soft $\alpha$-open sets (respectively, soft regular open sets, soft $\beta$-open sets) of a given soft anti-locally countable soft topological space and the soft $\alpha$-open sets (respectively, soft regular open sets, soft $\beta$-open sets) of the soft topological space of soft $\omega^{0}$-open sets generated by it. Finally, we introduce $\omega^{0}$-regularity in topological spaces via $\omega^{0}$-open sets, which is strictly between regularity and $\omega$-regularity, and we also introduce soft $\omega^{0}$-regularity in soft topological spaces via soft $\omega^{0}$-open sets, which is strictly between soft regularity and soft $\omega$-regularity. We investigate relationships regarding $\omega^{0}$-regularity and soft $\omega^{0}$-regularity. Moreover, we study the correspondence between soft $\omega^{0}$-regularity in soft topological spaces and $\omega^{0}$-regularity in topological spaces.
\end{abstract}

Keywords: soft $\omega$-open sets; $\omega^{0}$-open sets; soft anti-locally countable; soft regularity; soft $\omega$-regularity; soft generated soft topological spaces; soft induced topological spaces

\section{Introduction}

Some problems in medicine, engineering, the environment, economics, sociology, and other fields have their own doubts. Therefore, we are unable to deal with these problems by conventional methods. For more than thirty years, fuzzy set theory [1], rough set theory [2], and vague set theory [3] have played an essential role in dealing with these problems. Molodtsov [4] argues that each of these theories has its own set of problems. These difficulties mainly come from the inadequacy of the parameterization tool for the theories. Research through soft set theory has included almost all branches of science. Soft set theory has been applied to solve problems using Riemann integral, Beron's integral, game theory, function smoothness, operations research, measure theory, probability, and decision-making problems [5-10].

General topology, as one of the main branches of mathematics, is the branch of topology that deals with the basic definitions of set theory and structures used in topology. It is the foundation of most other branches of topology, including algebraic topology, geometric topology, and differential topology. Shabir and Naz [11] initiated soft topology, which is a new branch of topology that combines soft set theory and topology. Since that time, the generalization of topological concepts in soft topology has become the focus of many researchers, such as soft compact [12], soft connected [13], soft paracompact [13], soft extremely disconnected [14], soft Menger spaces [15], soft separable spaces [16], soft separation axioms [17-19], soft metric spaces [20-22], soft homogeneous spaces [23,24], and soft maps [25,26], and substantial contributions can still be made.

Generalizations of soft open sets play an effective role in soft topology through their use to improve on some known results or to open the door to redefine and investigate some 
of the soft topological concepts such as soft compactness, soft correlation, soft class axioms, soft assignments, etc. Some important generalizations of soft open sets appear in [27-34]. Soft $\omega$-open sets were defined and investigated by Al Ghour and Hamed [35], who used them to characterize soft Lindelofness and soft weakly Lindelofness. Then several research papers related to soft $\omega$-open sets were introduced [36,37]. The authors of [38] defined and investigated $\omega^{0}$-open sets as a generalization of open sets, which are a strong form of $\omega$-open sets. In the present paper, we extend $\omega^{0}$-open sets to include soft topological spaces. We investigate this class of soft sets, especially in soft Lindelof and soft anti-locally soft topological spaces. Additionally, via soft $\omega^{0}$-open sets, we introduce the class of soft $\omega^{0}$-regular spaces. This research will be of interest to many researchers, especially those who are interested in generalizing soft topological concepts or those who are interested in linking topology and soft topology.

The arrangement of this article is as follows:

In Section 2, we collect the main definitions and results that will be used in this research.

In Section 3, we define the class of soft $\omega^{0}$-open sets. With the help of examples, we show that this class of soft sets forms a soft topology that lies strictly between the soft topology of soft open sets and the soft topology of soft $\omega^{0}$-open sets, and we give some sufficient conditions for the equality between the three soft topologies. Additionally, we show that soft closed soft $\omega$-open sets are soft $\omega^{0}$-open sets in soft Lindelof soft topological spaces. In addition, we study the correspondence between soft $\omega^{0}$-open sets in soft topological spaces and $\omega^{0}$-open sets in topological spaces.

In Section 4, we study the correspondence between soft anti-locally countable soft topological spaces and anti-locally countable topological spaces. The relationships between the soft $\alpha$-open sets (respectively, soft regular open sets, soft $\beta$-open sets) of a given soft antilocally countable soft topological space and the soft $\alpha$-open sets (respectively, soft regular open sets, soft $\beta$-open sets) of the soft topological space of soft $\omega^{0}$-open sets generated by it are then investigated.

In Section 5, we introduce $\omega^{0}$-regularity in topological spaces via $\omega^{0}$-open sets, which is strictly between regularity and $\omega$-regularity. We also introduce soft $\omega^{0}$-regularity in soft topological spaces via soft $\omega^{0}$-open sets, which is strictly between soft regularity and soft $\omega$-regularity. We give several characterizations and relationships regarding $\omega^{0}$-regularity and soft $\omega^{0}$-regularity. Moreover, we study the correspondence between soft $\omega^{0}$-regularity in soft topological spaces and $\omega^{0}$-regularity in topological spaces.

In Section 6, we give some conclusions and possible future work.

\section{Preliminaries}

In this paper, we follow the notions and terminologies as they appear in $[23,34,37]$. Throughout this paper, ST and STS will denote topological space and soft topological space, respectively. Let $Y$ be an initial universe and $B$ be a set of parameters. In this paper, the family of all soft sets over $Y$ relative to $B$ will be denoted by $S S(Y, B)$. Let $(Y, \sigma, B)$ be a STS, $(Z, \mu)$ be a TS, $H \in S S(Y, B)$, and $S \subseteq Z$. Throughout this paper, $\sigma^{\mathcal{C}}$ will denote the collection of all soft closed sets of $(Y, \sigma, B)$, and $\mu^{\mathcal{C}}$ will denote the collection of all closed sets of $(Z, \mu)$, with $C l_{\sigma}(H), C l_{\mu}(S)$, Int $\sigma(H)$, and $\operatorname{Int}_{\mu}(S)$ denoting the soft closure of $H$ in $(Y, \sigma, B)$, the closure of $S$ in $(Z, \mu)$, the soft interior of $H$ in $(Y, \sigma, B)$, and the interior of $S$ in $(Z, \mu)$, respectively.

Now we recall some concepts that will be used in the sequel.

Definition 1. Let $(Y, \mu)$ be a TS and $S \subseteq Y$. Then

(a) a point $y \in Y$ is called a condensation point of $S$ if $V \cap S$ is uncountable for any $V \in \mu$ with $y \in V$;

(b) Ref. [39] $S$ is called an $\omega$-closed subset of $(Y, \mu)$ if $S$ contains all of its condensation points;

(c) Ref. [39] $S$ is called an $\omega$-open subset of $(Y, \mu)$ if $Y-S$ is an $\omega$-closed subset of $(Y, \mu)$;

(d) Ref. [39] The collection of all $\omega$-open subsets of $(Y, \mu)$ is denoted by $\mu_{\omega}$; 
(e) Ref. [38] $S$ is called an $\omega^{0}$-open set in $(Y, \mu)$ if for every $y \in S$, there exist $V \in \mu$ and a countable subset $A \subseteq Y$ such that $y \in V$ and $V-A \subseteq \operatorname{Int}_{\mu}(S)$;

(f) Ref. [38] $S$ is called an $\omega^{0}$-closed subset of $(Y, \mu)$ if $Y-S$ is an $\omega^{0}$-open subset of $(Y, \mu)$;

(g) Ref. [38] The collection of all $\omega^{0}$-open subsets of $(Y, \mu)$ will be denoted by $\mu_{\omega^{0}}$.

Theorem 1. For any $T S(Y, \mu)$ and any $S \subseteq Y$, we have:

(a) Ref. [39] $\left(Y, \mu_{\omega}\right)$ is a TS with $\mu \subseteq \mu_{\omega}$, and $\mu \neq \mu_{\omega}$ in general;

(b) Ref. [39] $S \in \mu_{\omega}$ if and only if for any $y \in S$ there exist $V \in \mu$ and a countable subset $A \subseteq Y$ such that $y \in V-A \subseteq S$;

(c) Ref. [38] $\left(Y, \mu_{\omega^{0}}\right)$ is a TS with $\mu \subseteq \mu_{\omega^{0}} \subseteq \mu_{\omega}, \mu \neq \mu_{\omega^{0}}$ in general, and $\mu_{\omega} \neq \mu_{\omega^{0}}$ in general;

(d) Ref. [38] $S \in \mu_{\omega^{0}}$ if and only if for every $y \in S$, there exists $V \in \mu$ such that $y \in V$ and $V-\operatorname{Int}_{\mu}(S)$ is countable.

Definition 2. A TS $(Y, \mu)$ is called

(a) Ref. [40] $\omega$-regular if for any $B \in \mu^{c}$ and $y \in Y-B$, there exist $U \in \mu$ and $V \in \mu_{\omega}$ such that $y \in U, B \subseteq V$ and $U \cap V=\varnothing$;

(b) Ref. [41] Anti-locally countable if each $V \in \mu-\{\varnothing\}$ is uncountable;

(c) Ref. [42] $\omega$-locally indiscrete if $\mu \subseteq\left(\mu_{\omega}\right)^{c}$.

Definition 3. Ref. [20] Let $Y$ be a universal set, $B$ be a set of parameters, and let $G \in S S(Y, B)$. If

$$
G(b)=\left\{\begin{array}{cl}
\{y\} & \text { if } b=e \\
\varnothing & \text { if } b \neq e
\end{array},\right.
$$

then $G$ is called a soft point in $S S(Y, B)$ and is denoted by $e_{y}$.

The set of all soft points in $S S(Y, B)$ will be denoted $S P(X, A)$.

Definition 4. Ref. [20] Let $G \in S S(Y, B)$ and $b_{y} \in S P(Y, B)$. Then, $b_{y}$ is said to belong to $G$ (notation: $\left.b_{y} \widetilde{\in} G\right)$ if $b_{y} \widetilde{\subseteq} G$, or equivalently: $b_{y} \widetilde{\in} G$ if and only if $y \in G(b)$.

Definition 5. Let $Y$ be a universal set and $B$ be a set of parameters. Then $G \in S S(Y, B)$ defined by

(a) Ref. [23] $G(b)=\left\{\begin{array}{ll}Z & \text { if } b=e \\ \varnothing & \text { if } b \neq e\end{array}\right.$ will be denoted by $e_{Z}$.

(b) Ref. [23] $G(b)=Z$ for all $b \in B$ will be denoted by $C_{Z}$.

Definition 6. A STS $(Y, \sigma, B)$ is called

(a) Ref. [43] A soft $T_{1}$ if for any $c_{y}, c_{z} \in S P(Y, B)$ with $y \neq z$, there exists $M, N \in \sigma$ such that $c_{y} \widetilde{\in} M-N$ and $c_{z} \widetilde{\in} N-M$;

(b) Ref. [43] $A$ soft $T_{2}$ if for any $c_{y}, d_{z} \in S P(Y, B)$ with $y \neq z$, we find $M, N \in \sigma$ such that $c_{y} \widetilde{\in} M, d_{z} \widetilde{\in} N$, and $M \widetilde{\cap} N=0_{B}$;

(c) Ref. [44] A soft $T_{2 \frac{1}{2}}$ if for any $c_{y}, d_{z} \in S P(Y, B)$ with $y \neq z$, we find $M, N \in \sigma$ such that $c_{y} \widetilde{\in} M, d_{z} \widetilde{\in} N$, and $C l_{\sigma}(M) \widetilde{\cap} C l_{\sigma}(N)=0_{B}$;

(d) Ref. [43] A soft regular if whenever $H \in \sigma^{c}$ and $b_{y} \widetilde{\in} 1_{B}-H$, then there exist $M, N \in \sigma$ such that $b_{y} \widetilde{\in} M, H \widetilde{\subseteq} N$, and $M \widetilde{\cap} N=0_{B}$.

Definition 7. Let $(Y, \sigma, B)$ be a STS and let $M \in S S(Y, B)$. Then, $M$ is called soft $\alpha$-open [28] (respectively, soft $\beta$-open [29], soft regular open [31]) in $(Y, \sigma, B)$ if $M \widetilde{\subseteq} \operatorname{Int}_{\sigma}\left(C l_{\sigma}\left(\operatorname{Int}_{\sigma}(M)\right)\right)$ (respectively, $M \subseteq C l_{\sigma}\left(\operatorname{Int}_{\sigma}\left(C l_{\sigma}(M)\right)\right), M=\operatorname{Int}_{\sigma}\left(C l_{\sigma}(M)\right)$ ). The families of soft $\alpha$-open sets, soft $\beta$-open sets, and soft regular open sets in $(Y, \sigma, B)$ are denoted, respectively, by $\alpha(Y, \sigma, B)$, $\beta(Y, \sigma, B)$, and $\operatorname{RO}(Y, \sigma, B)$.

Theorem 2. Ref. [11] Let $(Y, \sigma, B)$ be a STS. Then the collection $\{G(b): G \in \sigma\}$ defines a topology on $Y$ for every $b \in B$. This topology will be denoted by $\sigma_{b}$. 
Theorem 3. Ref. [45] Let $(Y, \mu)$ be a TS and and B be a set of parameters. Then the collection

$$
\{G \in S S(Y, B): G(b) \in \mu \text { for all } b \in B\}
$$

defines a soft topology on $Y$ relative to $B$. This soft topology will be denoted by $\tau(\mu)$.

Theorem 4. Ref. [23] Let $Y$ be an initial universe and let $B$ be a set of parameters. Let $\left\{\mu_{b}: b \in B\right\}$ be an indexed family of topologies on $Y$ and let

$$
\sigma=\left\{G \in S S(Y, B): G(b) \in \mu_{b} \text { for all } b \in B\right\} .
$$

Then $\sigma$ defines a soft topology on $Y$ relative to $B$. This soft topology will be denoted by $\underset{b \in B}{\oplus} \mu_{b}$.

\section{Soft $\omega^{0}$-Open Sets}

Definition 8. A soft set $M$ of a STS $(Y, \sigma, B)$ is called a soft $\omega^{0}$-open set in $(Y, \sigma, B)$ if for any $b_{y} \widetilde{\in} M$, we find $G \in \sigma$ and $K \in \operatorname{CSS}(Y, B)$ such that $b_{y} \widetilde{\in} G$ and $G-K \subseteq \operatorname{Int}_{\sigma}(M)$. Soft complements of soft $\omega^{0}$-open sets in $(Y, \sigma, B)$ are called soft $\omega^{0}$-closed sets in $(Y, \sigma, B)$.

The family of all soft $\omega^{0}$-open sets in $(Y, \sigma, B)$ will be denoted by $\sigma_{\omega^{0}}$.

Theorem 5. For any STS $(Y, \sigma, B), \sigma \subseteq \sigma_{\omega^{0}} \subseteq \sigma_{\omega}$.

Proof. For the inclusion $\sigma \subseteq \sigma_{\omega^{0}}$, let $M \in \sigma$ and $b_{y} \widetilde{\in} M$. If $K=0_{B}$, then $K \in \operatorname{CSS}(Y, B)$ and $b_{y} \widetilde{\in} M-K=M=\operatorname{Int}_{\sigma}(M) \widetilde{\widetilde{I n t}} t_{\sigma}(M)$. This shows that $M \in \sigma_{\omega^{0}}$. For the inclusion $\sigma_{\omega^{0}} \subseteq \sigma_{\omega}$, let $M \in \sigma_{\omega^{0}}$ and let $b_{y} \widetilde{\in} M$. Then we find $G \in \sigma$ and $K \in \operatorname{CSS}(Y, B)$ such that $b_{y} \widetilde{\in} G$ and $G-K \widetilde{\subseteq} \operatorname{Int}_{\sigma}(M) \widetilde{\subseteq} M$. This shows that $M \in \sigma_{\omega}$.

The following two examples will show that any of the inclusions in Theorem 5 cannot be replaced by equality, in general:

Example 1. Let $Y=\mathbb{R}, B=\mathbb{Z}$, $\mu$ be the usual topology on $Y$, and $\sigma=\{L \in S S(Y, B): L(b) \in \mu$ for every $b \in B\}$. Then, clearly, $C_{\mathbb{Q}^{c}} \in \sigma_{\omega}$. On the other hand, if $C_{\mathbb{Q}^{c}} \in \sigma_{\omega^{0}}$, then there exist $N \in \sigma$ and $K \in \operatorname{CSS}(Y, B)$ such that $1_{\pi} \widetilde{\in} N$ and $N-K \widetilde{\simeq} \operatorname{Int} t_{\sigma}\left(C_{\mathbb{Q}^{c}}\right)=0_{B}$, and so $N \widetilde{\widetilde{C}} K$. This implies that $N \in \operatorname{CSS}(Y, B)$ and so $\pi \in N(1) \in \mu$ which is impossible. It follows that $C_{\mathbb{Q}^{c}} \notin \sigma_{\omega^{0}}$.

Example 2. If $Y=\mathbb{Z}, B=\{a, b\}$, and $\sigma=\left\{0_{B}, 1_{B}\right\}$, then $C_{\mathbb{N}} \in \sigma_{\omega^{0}}-\sigma$.

Theorem 6. If $(Y, \sigma, B)$ is a soft $T_{1}$ and soft $p$-space, then $\sigma=\sigma_{\omega^{0}}=\sigma_{\omega}$.

Proof. Follows from Theorem 5 of this paper and Theorem 9 of [35].

Theorem 7. For any STS $(Y, \sigma, B),\left(Y, \sigma_{\omega^{0}}, B\right)$ is a STS.

Proof. According to Theorem 5, $\sigma \subseteq \sigma_{\omega^{0}}$, and so $0_{B}, 1_{B} \in \sigma_{\omega^{0}}$. Let $M, N \in \sigma_{\omega^{0}}$ and let $b_{y} \widetilde{\in} M \widetilde{\cap} N$. Then we have $b_{y} \widetilde{\in} M \in \sigma_{\omega^{0}}$ and $b_{y} \widetilde{\in} N \in \sigma_{\omega^{0}}$. Thus, we find $T, S \in \sigma$ and $K, L \in$ $\operatorname{CSS}(Y, B)$ such that $b_{y} \widetilde{\in} T, b_{y} \widetilde{\in} S, T-K \widetilde{\subseteq} \operatorname{Int} t_{\sigma}(M)$, and $S-L \subseteq \operatorname{Int}_{\sigma}(N)$. Hence, $b_{y} \widetilde{\in} T \widetilde{\cap} S \in$ $\sigma, K \widetilde{\cup} L \in \operatorname{CSS}(Y, B)$, and $(T \widetilde{\cap} S)-(K \widetilde{\cup} L) \widetilde{\subseteq}(T-K) \widetilde{\cap}(S-L) \widetilde{\subseteq} \operatorname{Int}_{\sigma}(M) \widetilde{\cap} \operatorname{Int} t_{\sigma}(N)=$ $\operatorname{Int}_{\sigma}(M \widetilde{\cap} N)$. Therefore, $M \widetilde{\cap} N \in \sigma_{\omega^{0}}$. Let $\left\{M_{\alpha}: \alpha \in \Gamma\right\} \subseteq \sigma_{\omega^{0}}$ and let $b_{y} \widetilde{\in} \cup_{\alpha \in \Gamma} M_{\alpha}$. Choose $\alpha_{\circ} \in \Gamma$ such that $b_{y} \widetilde{\in} M_{\alpha_{\circ}}$. Then there exist $S \in \sigma$ and $K \in \operatorname{CSS}(Y, B)$ such that $b_{y} \tilde{\in} S$ and $S-K \subseteq \tilde{I} I n t_{\sigma}\left(M_{\alpha_{\circ}}\right) \subseteq \widetilde{\subseteq} \operatorname{Int}_{\sigma}\left(\cup_{\alpha \in \Gamma} M_{\alpha}\right)$. Therefore, $\cup_{\alpha \in \Gamma} M_{\alpha} \in \sigma_{\omega^{0}}$.

Theorem 8. If $(Y, \sigma, B)$ is a STS such that $\left\{1_{B}-H: H \in \operatorname{CSS}(Y, B)\right\} \subseteq \sigma$, then $\sigma=\sigma_{\omega^{0}}=\sigma_{\omega}$.

Proof. Follows from Theorem 5 of this paper and Theorem 4 of [35].

Theorem 9. For any soft locally countable STS $(Y, \sigma, B),\left(Y, \sigma_{\omega^{0}}, B\right)$ is a discrete STS. 
Proof. Let $M \in S S(Y, B)-\left\{0_{B}\right\}$. Choose $b_{y} \widetilde{\in} M$. Since $(Y, \sigma, B)$ is soft locally countable, then there exists $N \in \operatorname{CSS}(Y, B) \cap \sigma$ such that $b_{y} \widetilde{\in} N \widetilde{\subseteq} M$. Thus, we have $b_{y} \widetilde{\in} N \in \sigma$, $N \in \operatorname{CSS}(Y, B)$ and $N-N=0_{B} \widetilde{\subseteq} \operatorname{Int}_{\sigma}(M)$. Therefore, $M \in \sigma_{\omega^{0}}$.

Corollary 1. Let $(Y, \sigma, B)$ be a STS such that $Y$ is a countable set. Then $\left(Y, \sigma_{\omega^{0}}, B\right)$ is a discrete STS.

Theorem 10. Let $(Y, \sigma, B)$ be a STS and $M \in S S(Y, B)$. Then $M \in \sigma_{\omega^{0}}$ if and only if for each $b_{y} \widetilde{\in} M$, there exists $N \in \sigma$ such that $b_{y} \widetilde{\in} N$ and $N-\operatorname{Int}_{\sigma}(M) \in \operatorname{CSS}(Y, B)$.

Proof. Necessity. Let $M \in \sigma_{\omega^{0}}$ and let $b_{y} \widetilde{\in} M$. Then we find $N \in \sigma$ and $K \in \operatorname{CSS}(Y, B)$ such that $b_{y} \widetilde{\in} N$ and $N-K \widetilde{\subseteq} \operatorname{Int}_{\sigma}(M)$. As $N-K \widetilde{\subseteq} \operatorname{Int}_{\sigma}(M)$, then $N-\operatorname{Int}_{\sigma}(M) \widetilde{\subseteq} K$. Thus, $N-\operatorname{Int}_{\sigma}(M) \in \operatorname{CSS}(Y, B)$.

Sufficiency. Suppose that for each $b_{y} \widetilde{\in} M$, there exists $N \in \sigma$ such that $b_{y} \widetilde{\in} N$ and $N-$ $\operatorname{Int}_{\sigma}(M) \in \operatorname{CSS}(Y, B)$. Let $b_{y} \widetilde{\in} M$. Then we find $N \in \sigma$ such that $b_{y} \tilde{\in} N$ and $N-\operatorname{Int}_{\sigma}(M) \in$ $\operatorname{CSS}(Y, B)$. If $K=N-\operatorname{Int}_{\sigma}(N)$, then $K \in \operatorname{CSS}(Y, B)$ and $N-K=\operatorname{Int}_{\sigma}(M) \widetilde{\subseteq} \operatorname{Int}_{\sigma}(M)$. Thus, $M \in \sigma_{\omega^{0}}$.

Theorem 11. Let $(Y, \sigma, B)$ be a STS. If $C_{Z} \in \sigma_{\omega^{0}}-\left\{0_{B}\right\}$, then $\left(\sigma_{\omega^{0}}\right)_{Z} \subseteq\left(\sigma_{Z}\right)_{\omega^{0}}$.

Proof. Let $N \in\left(\sigma_{\omega^{0}}\right)_{Z}$ and let $b_{z} \widetilde{\in} N$. Choose $M \in \sigma_{\omega^{0}}$ such that $N=M \widetilde{\cap} C_{Z}$. As $C_{Z} \in \sigma_{\omega^{0}}$, then $N \in \sigma_{\omega^{0}}$. Thus, we find $G \in \sigma$ and $K \in \operatorname{CSS}(Z, B)$ such that $b_{z} \widetilde{\in} G$ and $G-K$ $\widetilde{\subseteq} \operatorname{Int}_{\sigma}(N)$. Therefore, we have $b_{z} \widetilde{\in} G \widetilde{\cap} C_{Z} \in \sigma_{Z}, K \widetilde{\cap} C_{Z} \in \operatorname{CSS}(Z, B)$, and $\left(G \widetilde{\cap} C_{Z}\right)-$ $\left(K \widetilde{\cap} C_{Z}\right) \widetilde{\subseteq}(G-K) \widetilde{\cap} C_{Z} \widetilde{\subseteq} \operatorname{Int}_{\sigma}(N) \widetilde{\cap} C_{Z} \widetilde{\subseteq} \operatorname{Int} \sigma_{Z}(N)$.

Corollary 2. Let $(Y, \sigma, B)$ be a STS. If $C_{Z} \in \sigma-\left\{0_{B}\right\}$, then $\left(\sigma_{\omega^{0}}\right)_{Z} \subseteq\left(\sigma_{Z}\right)_{\omega^{0}}$.

As can be shown by the following example, the condition ' $C_{Y} \in \sigma_{\omega^{0}}$ ' is essential in Theorem 11.

Example 3. Let $Y=\mathbb{R}, Z=\mathbb{Q}^{c}, B=\{a, b, c\}, \mu$ be the usual topology on $Y$, and $\sigma=$ $\left\{C_{W}: W \in \mu\right\}$. Since $C_{(-\infty, 1)} \in \sigma \subseteq \sigma_{\omega^{0}}$, then $C_{(-\infty, 1)} \widetilde{n} C_{Z}=C_{(-\infty, 1) \cap \mathbb{Q}^{c}} \in\left(\sigma_{\omega^{0}}\right)_{Z}$. On the other hand, if $C_{(-\infty, 1) \cap \mathbb{Q}^{c}} \in\left(\sigma_{Z}\right)_{\omega^{0}}$, then there exists $W \in \mu$ and $K \in \operatorname{CSS}(Z, B)$ such that $a_{0} \in C_{W}$ and $C_{W}-K \widetilde{\subseteq} \operatorname{Int}_{\sigma_{Z}}\left(C_{(-\infty, 1) \cap \mathbb{Q}^{c}}\right)=0_{B}$. Thus, $C_{W} \widetilde{\widetilde{\subseteq}} K$, and hence $C_{W} \in \operatorname{CSS}(Z, B)$. Therefore, $W$ is a countable set and $W \in \mu-\{\varnothing\}$, which is impossible.

Theorem 12. Let $(Y, \sigma, B)$ be a STS. Then for each $b \in B,\left(\sigma_{\omega^{0}}\right)_{b} \subseteq\left(\sigma_{b}\right)_{\omega^{0}}$.

Proof. Let $b \in B$. Let $V \in\left(\sigma_{\omega^{0}}\right)_{b}$ and let $y \in V$. Choose $N \in \sigma_{\omega^{0}}$ such that $V=N(b)$. Since $b_{y} \widetilde{\in} N \in \sigma_{\omega^{0}}$, then we find $M \in \sigma$ and $K \in \operatorname{CSS}(Y, B)$ such that $b_{y} \widetilde{\in} M$ and $M-K \widetilde{\subseteq} \operatorname{Int}_{\sigma}(N)$. So, we have $y \in M(b) \in \sigma_{b}, K(b)$ is countable, and $M(b)-K(b) \subseteq\left(\operatorname{Int}_{\sigma}(N)\right)(b)$. On the other hand, $\left(\operatorname{Int}_{\sigma}(N)\right)(b) \subseteq \operatorname{Int}_{\sigma_{b}}(N(b))=\operatorname{Int}_{\sigma_{b}}(V)$. Therefore, $V \in\left(\sigma_{b}\right)_{\omega^{0}}$.

Corollary 3. Let $(Y, \sigma, B)$ be a STS. If $N \in \sigma_{\omega^{0}}$, then $N(b) \in\left(\sigma_{b}\right)_{\omega^{0}}$ for every $b \in B$.

Proof. Let $N \in \sigma_{\omega^{0}}$ and let $b \in B$. Then $N(b) \in\left(\sigma_{\omega^{0}}\right)_{b}$. Thus, by Theorem 12, $N(b) \in\left(\sigma_{b}\right)_{\omega^{0}}$.

Lemma 1. Let $Y$ be an initial universe and $B$ be a set of parameters. If $\left\{\left(Y, \mu_{b}\right): b \in B\right\}$ is a collection of TSs, then for every $c \in B$ and $V \in\left(\mu_{c}\right)_{\omega^{0}}, c_{I_{n t} \mu_{c}}(V) \widetilde{\subseteq} \underset{\operatorname{Int}}{\underset{b \in B}{\oplus} \mu_{b}}\left(c_{V}\right)$.

Proof. Let $c \in B$ and $V \in\left(\mu_{c}\right)_{\omega^{0}}$. Since $\operatorname{Int}_{\mu_{c}}(V) \in \mu_{c}$, then $c_{I n t_{\mu_{c}}(V)} \in \underset{b \in B}{\oplus} \mu_{b}$. Since $c_{I n t_{\mu_{c}}(V)} \widetilde{\subseteq} c_{V}$, then $c_{I n \mu_{\mu_{c}}(V)} \underset{\widetilde{I}}{\operatorname{Int}} \underset{b \in B}{\oplus \mu_{b}}\left(c_{V}\right)$. 
Theorem 13. Let $Y$ be an initial universe and $B$ be a set of parameters. If $\left\{\left(Y, \mu_{b}\right): b \in B\right\}$ is $a$ collection of TSs, then $\left(\underset{b \in B}{\oplus} \mu_{b}\right)_{\omega^{0}}=\underset{b \in B}{\oplus}\left(\mu_{b}\right)_{\omega^{0}}$.

Proof. By Theorem 12 of this paper and Theorem 8 of [23] we have $\left(\left(\underset{b \in B}{\oplus} \mu_{b}\right)_{\omega^{0}}\right)_{c} \subseteq$ $\left(\left(\underset{b \in B}{\oplus} \mu_{b}\right)_{c}\right)_{\omega^{0}}=\left(\mu_{c}\right)_{\omega^{0}}$ for every $c \in B$. Hence, $\left(\underset{b \in B}{\oplus} \mu_{b}\right)_{\omega^{0}} \subseteq \underset{b \in B}{\oplus}\left(\mu_{b}\right)_{\omega^{0}}$. To show that $\underset{b \in B}{\oplus}\left(\mu_{b}\right)_{\omega^{0}} \subseteq\left(\underset{b \in B}{\oplus} \mu_{b}\right)_{\omega^{0}}$, by Theorem 6 of [23], it is sufficient to show that $c_{V} \in\left(\underset{b \in B}{\oplus} \mu_{b}\right)_{\omega^{0}}$ for all $c \in B$ and $V \in\left(\mu_{c}\right)_{\omega^{0}}$. Let $c \in B$ and $V \in\left(\mu_{c}\right)_{\omega^{0}}$. Let $c_{y} \widetilde{\in} c_{V}$. Then we have $y \in V \in\left(\mu_{c}\right)_{\omega^{0}}$. Hence, there exist $W \in \mu_{c}$ and a countable subset $A \subseteq Y$ such that $y \in W$ and $W-A \subseteq \operatorname{Int}_{\mu_{c}}(V)$. Thus, we have $c_{y} \widetilde{\in} c_{W} \in \underset{b \in B}{\oplus} \mu_{b}, c_{A} \in \bar{C} S S(Y, B)$ and

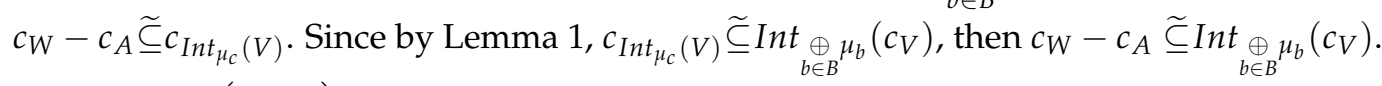
Hence, $c_{V} \in\left(\underset{b \in B}{\oplus} \mu_{b}\right)_{\omega^{0}}$.

Corollary 4. If $(Y, \mu)$ is a TS and B is any set of parameters, $(\tau(\mu))_{\omega^{0}}=\tau\left(\mu_{\omega^{0}}\right)$.

Proof. Let $\mu_{b}=\mu$ for all $b \in B$. Then $\tau(\mu)=\underset{b \in B}{\oplus} \mu_{b}$. Thus, by Theorem 13,

$$
\begin{aligned}
(\tau(\mu))_{\omega^{0}} & =\left(\bigoplus_{b \in B} \mu_{b}\right)_{\omega^{0}} \\
& =\underset{b \in B}{\oplus}\left(\mu_{b}\right)_{\omega^{0}} \\
& =\tau\left(\mu_{\omega^{0}}\right) .
\end{aligned}
$$

Theorem 14. If $(Y, \sigma, B)$ soft $T_{2}$, then $\left(Y, \sigma_{\omega^{0}}, B\right)$ is soft $T_{2}$.

Proof. Let $c_{y}, d_{z} \in S P(Y, B)$ such that $y \neq z$. As $(Y, \sigma, B)$ is a soft $T_{2}$, then we find $M, N \in \sigma \subseteq \sigma_{\omega^{0}}$ such that $b_{y} \widetilde{\in} M, b_{z} \widetilde{\in} N$, and $M \widetilde{\cap} N=0_{B}$. Therefore, $\left(Y, \sigma_{\omega^{0}}, B\right)$ is a soft $T_{2}$.

The following example will show that the converse of Theorem 14 is false:

Example 4. Let $Y=\mathbb{Z}, B=[0,1]$, and $\sigma=\left\{1_{B}, 0_{B}\right\}$. Then it is clear that $(Y, \sigma, B)$ is not a soft $T_{2}$. On the other hand, by Corollary $1,\left(Y, \sigma_{\omega^{0}}, B\right)$ is a soft $T_{2}$.

Theorem 15. Let $(Y, \sigma, B)$ be soft Lindelof. If $M \in \sigma_{\omega^{0}} \cap \sigma^{\mathcal{c}}$, then $M-\operatorname{Int} t_{\sigma}(M) \in \operatorname{CSS}(Y, B)$.

Proof. Let $M \in \sigma_{\omega^{0}} \cap \sigma^{\mathcal{c}}$. By Theorem 10, for every $b_{y} \widetilde{\in} M$, we find $N_{b_{y}} \in \sigma$ such that $b_{y} \widetilde{\in} N_{b_{y}}$ and $N_{b_{y}}-\operatorname{Int} t_{\sigma}(M) \in \operatorname{CSS}(Y, B)$. Set $\mathcal{A}=\left\{N_{b_{y}}: b_{y} \widetilde{\in} M\right\}$. Then $\mathcal{A}$ is a soft open cover of $M$. As $M \in \sigma^{\mathcal{C}}$ and $(Y, \sigma, B)$ is soft Lindelof, then there exists a countable subcover $\mathcal{A}_{1}$ of $\mathcal{A}$. Since $\widetilde{\cup}_{A \in \mathcal{A}_{1}}\left(A-\operatorname{Int} t_{\sigma}(M)\right) \in \operatorname{CSS}(Y, B)$ and $M-\operatorname{Int} t_{\sigma}(M) \widetilde{\subseteq}$ $\widetilde{\cup}_{A \in \mathcal{A}_{1}}\left(A-\operatorname{Int}_{\sigma}(M)\right)$, then $M-\operatorname{Int}_{\sigma}(M) \in \operatorname{CSS}(Y, B)$.

Corollary 5. Let $(Y, \sigma, B)$ be soft second countable. If $M \in \sigma_{\omega^{0}} \cap \sigma^{\mathcal{c}}$, then $M-\operatorname{Int}_{\sigma}(M) \in$ $\operatorname{CSS}(Y, B)$.

Theorem 16. If $(Y, \sigma, B)$ is a soft Lindelof STS, then $\sigma^{\mathcal{c}} \cap \sigma_{\omega} \subseteq \sigma_{\omega^{0}}$.

Proof. Suppose that $(Y, \sigma, B)$ is soft Lindelof. Let $M \in \sigma^{\mathcal{c}} \cap \sigma_{\omega}$ and let $b_{y} \widetilde{\in} M$. Then we find $N \in \sigma$ and $K \in \operatorname{CSS}(Y, B)$ such that $b_{y} \widetilde{\in} N$ and $N-K \widetilde{\subseteq} M$. Thus, $N-K-(M-$ 
$\left.\operatorname{Int}_{\sigma}(M)\right) \widetilde{\subseteq} M-\left(M-\operatorname{Int}_{\sigma}(M)\right)=\operatorname{Int}_{\sigma}(M)$. Since $K \in \operatorname{CSS}(Y, B)$ and by Theorem 5, $M-\operatorname{Int}_{\sigma}(M) \in \operatorname{CSS}(Y, B)$, then $K-\left(M-\operatorname{Int}_{\sigma}(M)\right) \in \operatorname{CSS}(Y, B)$. Hence, $M \in \sigma_{\omega^{0}}$.

Corollary 6. If $(Y, \sigma, B)$ is a soft second countable STS, then $\sigma^{c} \cap \sigma_{\omega} \subseteq \sigma_{\omega^{0}}$

Theorem 17. Let $(Y, \sigma, B)$ be STS. If $S \in\left(\sigma_{\omega}\right)^{c}$, then $S \widetilde{\subseteq} M \widetilde{\cup} K$ for some $M \in \sigma^{c}$ and $K \in$ $\operatorname{CSS}(Y, B)$.

Proof. Let $S \in\left(\sigma_{\omega}\right)^{c}$. If $S=1_{B}$, then $S \widetilde{\subseteq} 1_{B} \widetilde{\cup} 0_{B}$ such that $1_{B} \in \sigma^{c}$ and $0_{B} \in \operatorname{CSS}(Y, B)$. If $S \neq 1_{B}$, then we find $b_{y} \widetilde{\in} 1_{B}-S$. Since $1_{B}-S \in \sigma_{\omega}$, then we find $N \in \sigma$ and $K \in$ $\operatorname{CSS}(Y, B)$ such that $b_{y} \tilde{\in} N$ and $N-K \widetilde{\subseteq} 1_{B}-S$. Thus,

$$
\begin{aligned}
& S \widetilde{\subseteq} 1_{B}-(N-K) \\
& \widetilde{\subseteq} 1_{B}-\left(N \widetilde{\cap}\left(1_{B}-K\right)\right) \\
& \widetilde{\subseteq} 1_{B} \widetilde{\cap}\left(\left(1_{B}-N\right) \widetilde{\cup} K\right) \\
& \widetilde{\subseteq}\left(1_{B}-N\right) \widetilde{\cup} K .
\end{aligned}
$$

Let $M=1_{B}-N$. Then $M \in \sigma^{c}$ such that $S \widetilde{\subseteq} M \widetilde{\cup} K$.

Corollary 7. Let $(Y, \sigma, B)$ be STS. If $S \in\left(\sigma_{\omega^{0}}\right)^{c}$, then $S \widetilde{\subseteq} M \widetilde{\cup} K$ for some $M \in \sigma^{c}$ and $K \in$ $\operatorname{CSS}(Y, B)$.

Proof. Follows from Theorems 5 and 17.

\section{Soft Anti-Local Countability}

From now on, ALC and SALC will denote anti-locally countable and soft anti-locally countable, respectively.

Theorem 18. A STS $(Y, \sigma, B)$ is SALC if and only if $\left(Y, \sigma_{\omega^{0}}, B\right)$ is SALC.

Proof. Follows from Theorems 5 and 13 of [35].

Theorem 19. Let $(Y, \sigma, B)$ be a STS. If $\left(Y, \sigma_{b}\right)$ is ALC for every $b \in B$, then $(Y, \sigma, B)$ is SALC.

Proof. Suppose that $\left(Y, \sigma_{b}\right)$ is ALC for every $b \in B$. Let $M \in \sigma-\left\{0_{B}\right\}$. Choose $b_{y} \widetilde{\in} M$. Then $y \in M(b) \in \sigma_{b}$. Since $\left(Y, \sigma_{b}\right)$ is ALC, then $M(b)$ is uncountable. Hence, $M \notin \operatorname{CSS}(Y, B)$. It follows that $(Y, \sigma, B)$ is SALC.

Now we will give an example that shows that the opposite of Theorem 19 is not true in general.

Example 5. Let $Y=\mathbb{R}, B=\{a, b\}$, and $\sigma=\left\{0_{B}, 1_{B}, M\right\}$ where $M(a)=\mathbb{Q}$ and $M(b)=\mathbb{R}$. Then $(Y, \sigma, B)$ is SALC. On the other hand, since $\sigma_{a}=\{\varnothing, Y, \mathbb{Q}\}$, then $\left(Y, \sigma_{a}\right)$ is not ALC.

Theorem 20. Let $Y$ be an initial universe and let $B$ be a set of parameters. Let $\left\{\left(Y, \mu_{b}\right): b \in B\right\}$ be a collection of TSs. Then $\left(Y, \underset{b \in B}{\oplus} \mu_{b}, B\right)$ is SALC if and only if $\left(Y, \mu_{b}\right)$ is $A L C$ for each $b \in B$.

Proof. Necessity. Suppose that $\left(Y, \underset{b \in B}{\oplus} \mu_{b}, B\right)$ is SALC. Let $b \in B$ and let $V \in \mu_{b}-\{\varnothing\}$. Then $b_{V} \in \underset{b \in B}{\oplus} \mu_{b}$. Since $\left(Y, \underset{b \in B}{\oplus} \mu_{b}, B\right)$ is SALC, then $b_{V} \notin \operatorname{CSS}(Y, B)$. Since $b_{V}(c)=\varnothing$ for every $c \in B-\{b\}$, then $b_{V}(b)=V$ is uncountable. Hence, $(Y, \mu)$ is ALC. 
Sufficiency. Suppose that $(Y, \mu)$ is ALC for each $b \in B$. Let $\underset{b \in B}{\oplus} \mu_{b}=\sigma$. By Theorem 11 of [23], $\sigma_{b}=\left(\underset{b \in B}{\oplus} \mu_{b}\right)_{b}=\mu_{b}$ for each $b \in B$. Therefore, by Theorem 19, $\left(Y, \underset{b \in B}{\oplus} \mu_{b}, B\right)$ is SALC.

Corollary 8. For any TS $(Y, \mu)$ and any set of parameters $B,(Y, \mu)$ is ALC if and only if $(Y, \tau(\mu), B)$ is $S A L C$.

Proof. For each $b \in B$, set $\mu_{b}=\mu$. Then $\tau(\mu)=\underset{b \in B}{\oplus} \mu_{b}$. Thus, by Theorem 20 we get the result.

Theorem 21. If $(Y, \sigma, B)$ is $S A L C$ and $M \in \sigma_{\omega^{0}}$, then $C l_{\sigma}(M)=C l_{\omega^{0}}(M)$.

Proof. Suppose that $(Y, \sigma, B)$ is SALC and let $M \in \sigma_{\omega^{0}}$. Then clearly $C l_{\sigma_{\omega^{0}}}(M) \widetilde{\subseteq} C l_{\sigma}(M)$. We are going to show that $1_{B}-C l_{\omega_{\omega^{0}}}(M) \widetilde{\simeq} 1_{B}-C l_{\sigma}(M)$. Let $b_{y} \widetilde{\in} 1_{B}-C l_{\omega_{\omega^{0}}}(M) \in \sigma_{\omega^{0}}$. We find $N \in \sigma$ and $K \in \operatorname{CSS}(Y, B)$ such that $b_{y} \tilde{\in} N$ and $N-K \widetilde{\subseteq} \operatorname{Int}_{\sigma}\left(1_{B}-C l_{\omega_{\omega^{0}}}(M)\right) \widetilde{\subseteq} 1_{B}-$ $C l_{\omega_{\omega^{0}}}(M) \widetilde{\simeq} 1_{B}-M$. Thus, $N \widetilde{\cap} M \widetilde{\subseteq} K$ and hence $N \widetilde{\cap} M \in \operatorname{CSS}(Y, B)$. Since $N \widetilde{\cap} M \in \sigma_{\omega^{0}}$ and by Theorem $18,\left(Y, \sigma_{\omega^{0}}, B\right)$ is SALC, then $N \widetilde{\cap} M=0_{B}$. Therefore, we have $b_{y} \tilde{\in} N \in \sigma$ with $N \widetilde{\cap} M=0_{B}$, and hence $b_{y} \widetilde{\in} 1_{B}-C l_{\sigma}(M)$.

Corollary 9. If $(Y, \sigma, B)$ is SALC and $M$ is a soft $\omega^{0}$-closed set, then $\operatorname{Int}_{\sigma}(M)=\operatorname{Int}_{\sigma_{\omega^{0}}}(M)$.

As the following example shows, the condition "SALC" is necessary in Theorem 21.

Example 6. Let $Y=\mathbb{N}, B=\{a, b\}$ and $\sigma=\left\{0_{B}, 1_{B}, a_{1}\right\}$. Then $a_{1} \in \sigma \subseteq \sigma_{\omega^{0}}$ and $C l_{\sigma}\left(a_{1}\right)=1_{B}$. On the other hand, since $Y$ is countable, then by Corollary $1\left(Y, \sigma_{\omega^{0}}, B\right)$ is a discrete STS, and hence $C l_{\omega^{0} 0}\left(a_{1}\right)=a_{1} \neq 1_{B}$.

Theorem 22. For any SALC STS $(Y, \sigma, B), \alpha(Y, \sigma, B) \subseteq \alpha\left(Y, \sigma_{\omega^{0}}, B\right)$.

Proof. Let $(Y, \sigma, B)$ be SALC and let $M \in \alpha(Y, \sigma, B)$. Then $M \widetilde{\subseteq} \operatorname{Int}_{\sigma}\left(C_{\sigma}\left(\operatorname{Int}_{\sigma}(M)\right)\right)$. By Theorem 5, $\sigma \subseteq \sigma_{\omega^{0}}$. Thus, we have

$$
\begin{aligned}
& M \widetilde{\subseteq} \operatorname{Int}_{\sigma}\left(\operatorname{Cl}_{\sigma}\left(\operatorname{Int}_{\sigma}(M)\right)\right) \\
& \widetilde{\subseteq} \operatorname{Int}_{\sigma}\left(C l_{\sigma}\left(\operatorname{Int}_{\omega^{0}}(M)\right)\right) \\
& \widetilde{\subseteq} \operatorname{Int}_{\sigma_{\omega^{0}}}\left(\operatorname{Cl}_{\sigma}\left(\operatorname{Int}_{\omega_{\omega^{0}}}(M)\right)\right) .
\end{aligned}
$$

On the other hand, since $\operatorname{Int}_{\sigma_{\omega^{0}}}(M) \in \sigma_{\omega^{0}}$, then by Theorem 21, we have $C l_{\sigma}\left(\operatorname{Int}_{\sigma_{\omega^{0}}}(M)\right)=C l_{\omega^{0}}\left(\operatorname{Int}_{\sigma_{\omega^{0}}}(M)\right)$. Thus, $M \widetilde{\subseteq} \operatorname{Int}_{\sigma_{\omega^{0}}}\left(C l_{\sigma_{\omega^{0}}}\left(\operatorname{Int}_{\sigma_{\omega^{0}}}(M)\right)\right)$. Hence, $M \in$ $\alpha\left(Y, \sigma_{\omega^{0}}, B\right)$.

The inclusion in Theorem 22 cannot be replaced by equality in general, as will be shown in the following example:

Example 7. Consider the STS $(\mathbb{R}, \tau(\mu), \mathbb{N})$, where $\mu$ is the usual topology on $\mathbb{R}$. Since $(\mathbb{R}, \mu)$ is $A L C$, then by Corollary $8,(\mathbb{R}, \tau(\mu), \mathbb{N})$ is $S A L C$. On the other hand, it is not difficult to check that $C_{\mathbb{Q}^{c}} \in \alpha\left(\mathbb{R},(\tau(\mu))_{\omega^{0}}, \mathbb{N}\right)-\alpha(\mathbb{R}, \tau(\mu), \mathbb{N})$.

Theorem 23. For any $\operatorname{SALC} \operatorname{STS}(Y, \sigma, B), R O(Y, \sigma, B)=R O\left(Y, \sigma_{\omega^{0}}, B\right)$.

Proof. To show $R O(Y, \sigma, B) \subseteq R O\left(Y, \sigma_{\omega^{0}}, B\right)$, let $K \in R O(Y, \sigma, B)$. Then $K=\operatorname{Int}_{\sigma}\left(C l_{\sigma}(K)\right)$. Since $K \in \sigma \subseteq \sigma_{\omega^{0}}$, then by Theorem $21, C l_{\omega^{0}}(K)=C l_{\sigma}(K)$, and so $K=\operatorname{Int}_{\sigma}\left(C l_{\omega_{\omega^{0}}}(K)\right)$. 
On the other hand, since $C l_{\sigma}(K)$ is soft $\omega^{0}$-closed in $(Y, \sigma, B)$, then by Corollary 9 , $\operatorname{Int}_{\sigma}\left(C l_{\sigma_{\omega^{0}}}(K)\right)=\operatorname{Int}_{\sigma_{\omega^{0}}}\left(C l_{\sigma_{\omega^{0}}}(K)\right)=K$. Therefore, $K \in R O\left(Y, \sigma_{\omega^{0}}, B\right)$.

To show that $R O\left(Y, \sigma_{\omega^{0}}, B\right) \subseteq R O(Y, \sigma, B)$, let $K \in R O\left(Y, \sigma_{\omega^{0}}, B\right)$. Then $K=$ Int $_{\sigma_{\omega 0}}\left(C l_{\sigma^{0} 0}(K)\right)$. Since $C l_{\sigma^{0}}(K)$ is soft $\omega^{0}$-closed in $(Y, \sigma, B)$, then by Corollary 9, Int $\sigma_{\sigma^{0} 0}$ $\left(C l_{\omega^{0}}(K)\right)=\operatorname{Int}_{\sigma}\left(C l_{\omega^{0}}(K)\right)$. On the other hand, since $K \in \sigma \subseteq \sigma_{\omega^{0}}$, then by Theorem 21, $C l_{\omega^{0}}(K)=C l_{\sigma}(K)$. Therefore, $K=\operatorname{Int}_{\sigma}\left(C l_{\sigma}(K)\right)$. Hence, $K \in R O(Y, \sigma, B)$.

In Theorem 23, the condition in 'SALC' is essential, as the following example shows:

Example 8. Let $Y=\{1,2,3,4,5\}, \mu=\{\varnothing, Y,\{1\},\{1,2\},\{1,2,3\},\{1,2,3,4\}\}$. Consider the STS $(\mathbb{R}, \tau(\mu), \mathbb{N})$. Then $R O(\mathbb{R}, \tau(\mu), \mathbb{N})=\left\{0_{B}, 1_{B}\right\}$, while $R O\left(\mathbb{R},(\tau(\mu))_{\omega^{0}}, \mathbb{N}\right)=\tau(\mu)$.

Theorem 24. For any SALC STS $(Y, \sigma, B), \beta\left(Y, \sigma_{\omega^{0}}, B\right) \subseteq \beta(Y, \sigma, B)$.

Proof. Let $K \in \beta\left(Y, \sigma_{\omega^{0}}, B\right)$. Then $K \widetilde{\subseteq} C l_{\sigma_{\omega^{0}}}\left(\operatorname{Int}_{\sigma_{\omega^{0}}}\left(C l_{\omega_{\omega^{0}}}(K)\right)\right)$. Since $C l_{\omega_{\omega^{0}}}(K)$ is soft $\omega^{0}$-closed in $(Y, \sigma, B)$, then by Corollary 9 , Int $\operatorname{I}_{\omega^{0}}\left(C l_{\omega^{0}}(K)\right)=\operatorname{Int}_{\sigma}\left(C l_{\sigma^{0}}(K)\right)$. Thus, $K \subseteq \widetilde{\subseteq} C l_{\omega_{\omega^{0}}}\left(\operatorname{Int}_{\sigma}\left(C l_{\omega_{\omega^{0}}}(K)\right)\right)$. Additionally, since by Theorem $5, \sigma \subseteq \sigma_{\omega^{0}}$, then

$$
\begin{aligned}
& K \subseteq \widetilde{\subseteq} C l_{\sigma_{\omega^{0}}}\left(\operatorname{Int}_{\sigma}\left(C l_{\sigma^{0}}(K)\right)\right) \\
& \widetilde{\subseteq} C l_{\sigma^{0}}\left(\operatorname{Int}_{\sigma}\left(C l_{\sigma}(K)\right)\right) \\
& \widetilde{\subseteq} \operatorname{Int}_{\sigma}\left(C l_{\sigma}\left(\operatorname{Int}_{\sigma}(K)\right)\right) .
\end{aligned}
$$

Therefore, $K \in \beta(Y, \sigma, B)$.

Theorem 25. If $(Y, \sigma, B)$ is SALC and soft $T_{2 \frac{1}{2}}$, then $\left(Y, \sigma_{\omega^{0}}, B\right)$ is soft $T_{2 \frac{1}{2}}$.

Proof. Let $(Y, \sigma, B)$ be SALC and soft $T_{2 \frac{1}{2}}$. Let $b_{y}, c_{z} \in S P(Y, B)$ such that $y \neq z$. As $(Y, \sigma, B)$ is soft $T_{2 \frac{1}{2}}$, then we find $M, N \in \sigma$ with $C l_{\sigma}(M) \widetilde{\cap} C l_{\sigma}(N)=0_{B}$. Since by Theorem 5, $\sigma \subseteq \sigma_{\omega^{0}}$, then $M, N \in \sigma_{\omega^{0}}$. Additionally, by Theorem 21, we have $C l_{\sigma}(M)=C l_{\omega^{0}}(M)$ and $C l_{\sigma}(N)=C l_{\omega^{0}}(N)$. Hence, $C l_{\omega_{\omega^{0}}}(M) \widetilde{\cap} C l_{\omega_{\omega^{0}}}(N)=0_{B}$. Therefore, $\left(Y, \sigma_{\omega^{0}}, B\right)$ is a soft $T_{2 \frac{1}{2}}$.

\section{Soft $\omega^{0}$-Regularity}

Definition 9. A TS $(Y, \mu)$ is called $\omega^{0}$-regular if given any point $y \in Y$ and $L \in \mu^{c}$ such that $y \in Y-L$, there exist $S \in \mu$ and $T \in \mu_{\omega^{0}}$ such that $y \in S, L \subseteq T$, and $S \cap T=\varnothing$.

Theorem 26. $A$ TS $(Y, \mu)$ is $\omega^{0}$-regular if given any $S \in \mu$ and $y \in S$, there exists $V \in \mu$ such that $y \in V \subseteq C l_{\mu_{\omega^{0}}}(V) \subseteq S$.

Proof. Necessity. Suppose that $(Y, \mu)$ is soft $\omega^{0}$-regular. Let $S \in \mu$ and $y \in S$. Then $Y-S \in \mu^{c}$ and $y \in Y-(Y-S)$. Since $(Y, \mu)$ is $\omega^{0}$-regular, then there exist $V \in \mu$ and $W \in \mu_{\omega^{0}}$ such that $y \in V, Y-S \subseteq W$, and $V \cap W=\varnothing$. Since $Y-W \in\left(\mu_{\omega^{0}}\right)^{c}$ and $V \subseteq Y-W$, then $y \in V \subseteq C l_{\mu_{\omega^{0}}}(V) \subseteq Y-W \subseteq S$.

Sufficiency. Suppose that whenever $S \in \mu$ and $y \in S$, then there exists $V \in \mu$ such that $y \in V \subseteq C l_{\mu_{\omega^{0}}}(V) \subseteq S$. Let $L \in \mu^{c}$ and $y \in Y-L \in \mu$. Then there exists $T \in \mu$ such that $y \in T \subseteq C l_{\mu^{0} 0}(T) \subseteq Y-L$. Let $V=Y-C l_{\omega^{0} 0}(T)$. Then $V \in \mu_{\omega^{0}}$ and $L \subseteq V$. Therefore, $(Y, \mu)$ is $\omega^{0}$-regular.

Theorem 27. Locally countable TSs are $\omega^{0}$-regular. 
Proof. Let $(Y, \mu)$ be locally countable. Let $S \in \mu$ and $y \in S$. Since $(Y, \mu)$ is locally countable, then $\left(Y, \mu_{\omega^{0}}\right)$ is a discrete TS (see [38]). Therefore, $S=C l_{\mu_{\omega^{0}}}(S)$. Hence, we have $S \in \mu$ and $y \in S \subseteq C l_{\mu^{0}}(S) \subseteq S$. Therefore, by Theorem 26, $(Y, \mu)$ is $\omega^{0}$-regular.

Theorem 28. Every regular TS is $\omega^{0}$-regular.

Proof. Let $(Y, \mu)$ be a regular TS. Let $S \in \mu$ and $y \in S$. Since $(Y, \mu)$ is regular, then there exists $V \in \mu$ such that $y \in V \subseteq C l_{\mu_{\omega^{0}}}(V) \subseteq C l_{\mu}(V) \subseteq S$. Therefore, $(Y, \mu)$ is $\omega^{0}$-regular.

The following is an example of an $\omega^{0}$-regular TS that is not regular:

Example 9. Let $Y=\mathbb{Q}$ and $\mu=\{\varnothing\} \cup\{S \subseteq Y: Y-S$ is finite $\}$. Then $(Y, \mu)$ is not regular, while by Theorem $27,(Y, \mu)$ is $\omega^{0}$-regular.

Theorem 29. Every ALC $\omega^{0}$-regular TS is regular.

Proof. Let $(Y, \mu)$ be ALC and $\omega^{0}$-regular. Let $S \in \mu$ and $y \in S$. Since $(Y, \mu)$ is $\omega^{0}$ regular, then there exists $V \in \mu$ such that $y \in V \subseteq C l_{\mu_{\omega 0}}(V) \subseteq S$. Since $(Y, \mu)$ is $\operatorname{ALC}$, then $C l_{\mu^{0}}(V)=C l_{\mu}(V)$ (see [38]). Therefore, $y \in V \subseteq C l_{\mu}(V) \subseteq S$. Hence, $(Y, \mu)$ is regular.

Theorem 30. Every $\omega^{0}$-regular TS is $\omega$-regular.

Proof. Let $(Y, \mu)$ be $\omega^{0}$-regular. Let $S \in \mu$ and $y \in S$. Since $(Y, \mu)$ is $\omega^{0}$-regular, then there exists $V \in \mu$ such that $y \in V \subseteq C l_{\mu^{0} 0}(V) \subseteq S$. Since by Theorem $5, \sigma_{\omega^{0}} \subseteq \sigma_{\omega}$, then $C l_{\mu_{\omega}}(V) \subseteq C l_{\mu_{\omega^{0}}}(V)$. Therefore, $y \in V \subseteq C l_{\mu_{\omega}}(V) \subseteq C l_{\mu_{\omega^{0}}}(V) \subseteq S$. Hence, $(Y, \mu)$ is $\omega$-regular.

The following is an example of an $\omega$-regular TS that is not $\omega^{0}$-regular:

Example 10. Let $Y=\mathbb{R}$ and $\mu=\{\varnothing, Y, \mathbb{N}\}$. Since $(Y, \mu)$ is $\omega$-locally indiscrete, then by Corollary 15 of [42], $(Y, \mu)$ is $\omega$-regular.

Claim 1. $(Y, \mu)$ is not $\omega^{0}$-regular.

Proof of Claim 1. Suppose to the contrary that $(Y, \mu)$ is $\omega^{0}$-regular. Then by Theorem 26, there exists $V \in \mu$ such that $1 \in V \subseteq C l_{\mu_{\omega 0}}(V) \subseteq \mathbb{N}$. Therefore, $V=$ $\mathbb{N}=\mathrm{Cl}_{\mu_{\omega^{0}}}(\mathbb{N})$. Thus, $\mathbb{R}-\mathbb{N} \in \mu_{\omega^{0}}$ and so we find $T \in \mu$ and a countable set $D \subseteq \mathbb{R}$ such that $-1 \in T$ and $T-D \subseteq \operatorname{Int}_{\mu}(\mathbb{R}-\mathbb{N})=\varnothing$. Therefore, we have $T=\mathbb{R} \subseteq D$, a contradiction.

Definition 10. A STS $(Y, \sigma, B)$ is called soft $\omega^{0}$-regular if given any $b_{y} \widetilde{\in} S P(Y, B)$ and $R \in \sigma^{c}$ such that $b_{y} \widetilde{\in} 1_{B}-R$, there exist $S \in \sigma$ and $T \in \sigma_{\omega^{0}}$ such that $b_{y} \widetilde{\in} S, R \widetilde{\subseteq} T$, and $S \widetilde{\cap} T=0_{B}$.

Theorem 31. For any STS $(Y, \sigma, B)$, the following are equivalent:

(a) $(Y, \sigma, B)$ is soft $\omega^{0}$-regular;

(b) For any $b_{y} \in S P(Y, B)$ and any $W \in \sigma$ such that $b_{y} \widetilde{\in} W$, there exists $K \in \sigma$ such that $b_{y} \tilde{\in} K \widetilde{\subseteq} C l_{\omega^{0}}(K) \widetilde{\widetilde{\subseteq}} W$.

Proof. (a) $\Longrightarrow$ (b): Let $b_{y} \in S P(Y, B)$ and $W \in \sigma$ such that $b_{y} \tilde{\in} W$. Then $1_{B}-W \in \sigma^{c}$ and $b_{y} \widetilde{\in} 1_{B}-\left(1_{B}-W\right)$. By (a), we find $K \in \sigma$ and $S \in \sigma_{\omega^{0}}$ such that $b_{y} \widetilde{\in} K, 1_{B}-W \widetilde{\subseteq} S$ and $K \widetilde{\cap} S=0_{B}$. Therefore, $b_{y} \widetilde{\in} K \widetilde{\subseteq} C l_{\omega_{\omega^{0}}}(K) \widetilde{\widetilde{\subseteq}} 1_{B}-S \widetilde{\subseteq} W$. 
(b) $\Longrightarrow$ (a): Let $b_{y} \in S P(Y, B)$ and $L \in \sigma^{c}$ such that $b_{y} \widetilde{\in} 1_{B}-L \in \sigma$. Then by (b), we find $K \in \sigma$ such that $b_{y} \widetilde{\in} K \widetilde{\subseteq} C l_{\omega_{\omega^{0}}}(K) \widetilde{\widetilde{\subseteq}} 1_{B}-L$. Let $S=1_{B}-C l_{\omega_{\omega^{0}}}(K)$. Then $S \in \sigma_{\omega^{0}}$ and $K \widetilde{\subseteq}$. This shows that $(Y, \sigma, B)$ is soft $\omega^{0}$-regular.

Theorem 32. Soft locally countable STSs are soft $\omega^{0}$-regular STSs.

Proof. Follows from Theorems 9 and 31.

Theorem 33. Soft regular STSs are soft $\omega^{0}$-regular STSs.

Proof. Let $(Y, \sigma, B)$ be soft regular. Let $b_{y} \in S P(Y, B)$ and $W \in \sigma$ such that $b_{y} \tilde{\in} W$. We find $S \in \sigma$ such that $b_{y} \widetilde{\in} S \widetilde{\subseteq} C l_{\sigma}(S) \widetilde{\subseteq} W$. As $\sigma \subseteq \sigma_{\omega^{0}}$, then $C l_{\omega_{\omega^{0}}}(S) \widetilde{\subseteq} C l_{\sigma}(S)$. Thus, $b_{y} \tilde{\in} S \widetilde{\subseteq} C l_{\omega^{0}}(S) \widetilde{\subseteq} C l_{\sigma}(S) \widetilde{\subseteq} W$. Therefore, $(Y, \sigma, B)$ is soft $\omega^{0}$-regular.

Theorem 34. Soft $\omega^{0}$-regular STSs are soft $\omega$-regular STSs.

Proof. Let $(Y, \sigma, B)$ be soft $\omega^{0}$-regular. Let $b_{y} \in S P(Y, B)$ and $W \in \sigma$ such that $b_{y} \widetilde{\in} W$. We find $S \in \sigma$ such that $b_{y} \widetilde{\in} S \subseteq \widetilde{\subseteq} C l_{\sigma^{0}}(S) \subseteq \widetilde{\subseteq} W$. As $\sigma_{\omega^{0}} \subseteq \sigma_{\omega}$, then $C l_{\sigma_{\omega}}(S) \subseteq \widetilde{\subseteq} C l_{\sigma^{0}}(S)$. Thus, $b_{y} \tilde{\in} S \subseteq \widetilde{\subseteq} C l_{\sigma_{\omega}}(S) \widetilde{\subseteq} C l_{\sigma^{0}}(S) \widetilde{\subseteq} W$. Therefore, $(Y, \sigma, B)$ is soft $\omega$-regular.

Theorem 35. If $(Y, \sigma, B)$ is soft $\omega^{0}$-regular, then $\left(Y, \sigma_{b}\right)$ is $\omega^{0}$-regular for every $b \in B$.

Proof. Let $b \in B$. Let $y \in Y$ and let $W \in \sigma_{b}$ such that $y \in W$. Pick $S \in \sigma$ such that $S(b)=W$. Since $b_{y} \widetilde{\in} S \in \sigma$ and $(Y, \sigma, B)$ is soft $\omega^{0}$-regular, then we find $T \in \sigma$ such that $b_{y} \tilde{\in} T \widetilde{\subseteq} C l_{\sigma_{\omega^{0}}}(T) \widetilde{\subseteq} S$, and thus, $y \in T(b) \subseteq\left(C l_{\omega_{\omega^{0}}}(T)\right)(b) \subseteq S(b)=W$. By Theorem 12, we have $T(b) \subseteq \mathrm{Cl}_{\left(\sigma_{b}\right)_{\omega^{0}}}(T(b)) \subseteq C l_{\left(\sigma_{\omega^{0}}\right)_{b}}(T(b))$. Additionally, by Proposition 7 of [11], we have $C l_{\left(\sigma_{\omega^{0}}\right)_{b}}(T(b)) \subseteq\left(C l_{\omega^{0}}(T)\right)(b)$. Therefore, we have $T(b) \in \sigma_{b}$, and $y \in T(b) \subseteq$ $C l_{\left(\sigma_{b}\right)_{\omega^{0}}}(T(b)) \subseteq S(b)=W$. It follows that $\left(Y, \sigma_{b}\right)$ is $\omega^{0}$-regular.

Theorem 36. Let $\left\{\left(Y, \mu_{b}\right): b \in B\right\}$ be a collection of STSs. Then $\left(Y, \underset{b \in B}{\oplus} \mu_{b}, B\right)$ is soft $\omega^{0}$-regular if and only if $\left(Y, \mu_{b}\right)$ is $\omega^{0}$-regular for every $b \in B$.

Proof. Necessity. Let $\left(Y, \underset{b \in B}{\oplus} \mu_{b}, B\right)$ be soft $\omega^{0}$-regular and let $c \in B$. Then by Theorem 36, $\left(Y,\left(\underset{b \in B}{\oplus} \mu_{b}\right)_{c}\right)$ is $\omega^{0}$-regular. Furthermore, by Theorem 8 of [23], $\left.\underset{b \in B}{\oplus} \mu_{b}\right)_{c}=\mu_{c}$. Therefore, $\left(Y, \mu_{c}\right)$ is $\omega^{0}$-regular.

Sufficiency. Let $\left(Y, \mu_{b}\right)$ be $\omega^{0}$-regular for every $b \in B$. Let $\sigma=\underset{b \in B}{\oplus} \mu_{b}$. Let $K \in \sigma$ and let $c_{y} \widetilde{\in} K$. Then $y \in K(c) \in \mu_{c}$. Since $\left(Y, \mu_{c}\right)$ is $\omega^{0}$-regular, then there exists $W \in \mu_{c}$ such that $y \in W \subseteq C l_{\left(\mu_{c}\right)_{\omega^{0}}}(W) \subseteq K(c)$. Thus, we have $c_{y} \widetilde{\in} c_{W} \widetilde{\subseteq} c_{C l_{\left(\mu_{c}\right)^{0}}}(V)=\widetilde{\subseteq} K$ with $c_{W} \in \sigma$. Additionally, by Theorem 13, we have $\sigma_{\omega^{0}}=\left(\underset{b \in B}{\oplus} \mu_{b}\right)_{\omega^{0}}=\underset{b \in B}{\oplus}\left(\mu_{b}\right)_{\omega^{0}}$. Hence, by Proposition 2 of [37], $c_{C l_{\left(\mu_{c}\right)_{\omega^{0}}}(W)}=C l_{\omega_{\omega^{0}}}\left(c_{W}\right)$. This shows that $\left(Y, \underset{b \in B}{\oplus} \mu_{b}, B\right)$ is soft $\omega^{0}$-regular.

Corollary 10. For any $T S(Y, \mu)$ and any set of parameters $B,(Y, \tau(\mu), B)$ is soft $\omega^{0}$-regular if and only if $(Y, \mu)$ is $\omega^{0}$-regular.

Proof. For every $b \in B$, let $\mu_{b}=\mu$. Then $\tau(\mu)=\underset{b \in B}{\oplus} \mu_{b}$. The result follows from Theorem 37. 
Now we give an example to show that the converse of Theorem 33 need not be true, in general:

Example 11. Let $(Y, \mu)$ be as in Example 8 and $B$ be any set of parameters. Then $(Y, \mu)$ is $\omega^{0}$-regular but not regular. Thus, by Corollary 10 and Corollary 4 of [37], $(Y, \tau(\mu), B)$ is soft $\omega^{0}$-regular but not soft regular.

Now we give an example to show that the converse of Theorem 35 need not be true, in general:

Example 12. Let $(Y, \mu)$ be as in Example 9 and $B$ be any set of parameters. Then $(Y, \mu)$ is $\omega$ regular but not $\omega^{0}$-regular. Thus, by Corollary 5 of [37] and Corollary 10, $(Y, \tau(\mu), B)$ is soft $\omega$-regular but not soft $\omega^{0}$-regular.

The following example shows that the implication in Theorem 36 is not reversible, in general:

Example 13. Let $(X, \tau, A)$ be as in Example 7 of [37]. It is proved in [37] that $\left(X, \tau_{a}\right)$ and $\left(X, \tau_{b}\right)$ are regular TSs. Thus, they are $\omega^{0}$-regular TSs. On the other hand, it is proved in [37] that $(X, \tau, A)$ is not soft $\omega$-regular, and so $(X, \tau, A)$ is not soft $\omega^{0}$-regular.

Theorem 37. If $(Y, \sigma, B)$ is a soft $\omega^{0}$-regular STS and $C_{Z} \in \sigma-\left\{0_{B}\right\}$, then $\left(Z, \sigma_{Z}, B\right)$ is soft $\omega^{0}$-regular.

Proof. Let $M \in\left(\sigma_{Z}\right)^{c}$ and $b_{z} \widetilde{\in} C_{Z}-M$. Choose $N \in \sigma^{c}$ such that $M=N \widetilde{\cap} C_{Z}$. As $N \in$ $\sigma^{\mathcal{C}}, b_{z} \widetilde{\in} 1_{B}-N$, and $(Y, \sigma, B)$ is soft $\omega^{0}$-regular, we find $T \in \sigma$ and $S \in \sigma_{\omega^{0}}$ such that $b_{z} \widetilde{\in} T, N \widetilde{\subseteq} S$ and $T \widetilde{\cap} S=0_{B}$. Therefore, $b_{z} \widetilde{\in} T \widetilde{\cap} C_{Z} \in \sigma_{Z}, M=N \widetilde{\cap} C_{Z} \widetilde{\subseteq} S \widetilde{\cap} C_{Z}$ with $S \widetilde{\cap} C_{Z} \in$ $\left(\sigma_{\omega^{0}}\right)_{Z}$, and $\left(T \widetilde{\cap} C_{Z}\right) \widetilde{\cap}\left(S \widetilde{\cap} C_{Z}\right)=(T \widetilde{\cap} S) \widetilde{\cap} C_{Z}=0_{B} \widetilde{\cap} C_{Z}=0_{B}$. Moreover, by Corollary 2, $S \widetilde{\cap} C_{Z} \in\left(\sigma_{Z}\right)_{\omega^{0}}$.

\section{Conclusions}

The growth of topology has been supported by the continuous supply of topological space classes, examples, properties, and relationships. As a result, expanding the structure of soft topological spaces in the same way is important.

In this paper, we introduce soft $\omega^{0}$-open sets as a new generalization of soft open sets. We have proved that the family of soft $\omega^{0}$-open sets forms a soft topology that lies between the soft topologies of soft open sets and soft $\omega$-open sets. Several relationships and connections to other well-known "generalized soft open" sets are given. Then, we have employed $\omega^{0}$-open sets and soft $\omega^{0}$-open sets to define $\omega^{0}$-regularity and soft $\omega^{0}$-regularity, respectively. We have characterized and investigated the main features of each of these two notions. In addition, we study the correspondence between our new soft topological notions and their analogous topological ones.

In the upcoming work, we plan to: (1) introduce some soft topological concepts using soft $\omega^{0}$-open sets such as soft connectedness, soft compactness, soft Lindelofness, soft separation axioms, and soft continuity; (2) introduce a decomposition of soft continuity; and (3) investigate the behavior of soft $\omega^{0}$-open sets as well as soft $\omega^{0}$-regularity under product soft topological spaces.

Funding: This research received no external fund.

Institutional Review Board Statement: Not applicable.

Informed Consent Statement: Not applicable.

Data Availability Statement: Not applicable.

Conflicts of Interest: The author declares no conflict of interest. 


\section{References}

1. Zadeh, L.A. Fuzzy sets. Inf. Control 1965, 8, 338-353. [CrossRef]

2. Pawlak, Z. Rough sets. Int. J. Comput. Inf. Sci. 1982, 11, 341-356. [CrossRef]

3. Gau, W.L.; Buehrer, D.J. Vague sets. IEEE Trans. Syst. Man Cybern. 1993, 23, 610-614. [CrossRef]

4. Molodtsov, D. Soft set theory-First results. Global optimization, control, and games, III. Comput. Math. Appl. 1999, 37, 19-31. [CrossRef]

5. Maji, P.K.; Roy, A.R.; Biswas, R. An application of soft sets in a decision making problem. Comput. Math. Appl. 2002, 44, 1077-1083. [CrossRef]

6. Molodtsov, D.; Leonov, V.Y.; Kovkov, D.V. Soft sets technique and its application. Nechetkie Sist. I Myagkie Vychisleniya 2006, 1,8-39.

7. Ali, G.; Alolaiyan, H.; Pamučar, D.; Asif, M.; Lateef, N. A Novel MADM Framework under q-Rung Orthopair Fuzzy Bipolar Soft Sets. Mathematics 2021, 9, 2163. [CrossRef]

8. Ali, G.; Afzal, M.; Asif, M.; Shazad, A. Attribute reduction approaches under interval-valued $q$-rung orthopair fuzzy soft framework. Appl. Intell. 2021. [CrossRef]

9. Akram, M.; Ali, G.; Peng, X.; Abidin, M.Z.U. Hybrid group decision-making technique under spherical fuzzy N-soft expert sets. Artif. Intell. Rev. 2021. [CrossRef]

10. Akram, M.; Ali, G.; Alcantud, J.C.R.; Riaz, A.Group decision-making with Fermatean fuzzy soft expert knowledge. Artif. Intell. Rev. 2022. [CrossRef]

11. Shabir, M.; Naz, M. On soft topological spaces. Comput. Math. Appl. 2011, 61, 1786-1799. [CrossRef]

12. Aygunoglu, A.; Aygun, H. Some notes on soft topological spaces. Neural Comput. Appl. 2012, 21, 113-119. [CrossRef]

13. Lin, F. Soft connected spaces and soft paracompact spaces. Int. J. Math. Comput. Sci. 2013, 7, $277-283$.

14. Asaad, B.A. Results on soft extremely disconnectedness of soft topological spaces. J. Math. Comput. Sci. 2017, 17, 448-464. [CrossRef]

15. Al-Shami, T.M.; Kocinac, L.D. Nearly soft menger spaces. J. Math. 2020, 2020, 3807418. [CrossRef]

16. Bayramov, S.; Gunduz, C. A new approach to separability and compactness in soft topological spaces. TWMS J. Pure Appl. Math. 2018, 9, 82-93.

17. Al-shami, T.M.; El-Shafei, M.E. Partial belong relation on soft separation axioms and decision-making problem, two birds with one stone. Soft Comput. 2020, 24, 5377-5387. [CrossRef]

18. El-Shafei, M.E.; Al-shami, T.M. Applications of partial belong and total non-belong relations on soft separation axioms and decision-making problem. Comput. Appl. Math. 2020, 39, 138. [CrossRef]

19. Al-shami, T.M. On Soft Separation Axioms and Their Applications on Decision-Making Problem. Math. Probl. Eng. 2021, 2021, 8876978. [CrossRef]

20. Das, S.; Samanta, S.K. Soft metric. Ann. Fuzzy Math. Inform. 2013, 6, 77-94

21. Çetkin, V.; Güner, E.; Aygün, H. On 2S-metric spaces. Soft Comput. 2020, 24, 12731-12742. [CrossRef]

22. Badyakar, U.; Nazmul, S. Some fixed soft point results on soft S-metric spaces. Math. Sci. 2021, 15, 283-291. [CrossRef]

23. Al Ghour, S.; Bin-Saadon, A. On some generated soft topological spaces and soft homogeneity. Heliyon 2019, 5, e02061. [CrossRef] [PubMed]

24. Matejdes, M. Soft homogeneity of soft topological sum. Soft Comput. 2021, 25, 8875-8881. [CrossRef]

25. Al-shami, T.M.; Alshammari, I.; Asaad, B.A. Soft maps via soft somewhere dense sets. Filomat 2020, 34, 3429-3440. [CrossRef]

26. Al-Shami, T.M. Defining and investigating new soft ordered maps by using soft semi open sets. Acta Univ. Sapientiae Math.2021, 13, 145-163. [CrossRef]

27. Chen, B. Soft semi-open sets and related properties in soft topological spaces. Appl. Math. Inf. Sci. 2013, 7, 287-294. [CrossRef]

28. Akdag, M.; Ozkan, A. Soft $\alpha$-open sets and soft $\alpha$-continuous functions. Abstr. Appl. Anal. 2014, 2014, 891341. [CrossRef]

29. Akdag, M.; Ozkan, A. Soft $\beta$-open sets and soft $\beta$-continuous functions. Sci. World J. 2014, 2014, 843456. [CrossRef]

30. Evanzalin Ebenanjar, P.; Thangavelu, P. Between nearly open sets and soft nearly open sets. Appl. Math. Inform. Sci. 2016, 10, 2277-2281. [CrossRef]

31. Hussain, S. On soft regular (closed) sets in soft topological spaces. J. Appl. Math. Inform. 2018, 36, 59-68.

32. Al-Shami, T.M. Soft somewhere dense sets on soft topological spaces. Commun. Korean Math. Soc. 2018, 33, 1341-1356.

33. Gaur, M.; Gupta, M.K. Soft $\theta$-generalized closed sets with respect to a soft ideal. J. Interdiscip. 2020, 23, 1241-1249. [CrossRef]

34. Kandemir, M.B. Some notes on $\sigma$-algebraic soft sets. Ann. Univ. Craiova Math. Comput. Ser. 2020, 47, 387-396.

35. Al Ghour, S.; Hamed, W. On two classes of soft sets in soft topological spaces. Symmetry 2020, 12, 265. [CrossRef]

36. Granados, C. On soft $b$-open sets. J. Indonesian Math. Soc. 2021, 27, 123-129. [CrossRef]

37. Al Ghour, S. Weaker forms of soft regular and soft $T_{2}$ soft topological spaces. Mathematics 2021, 9, 2153. [CrossRef]

38. Al-Hawary, T.; Al-Omari, A. Between open and $\omega$-open sets. Quest. Answers Gen. Topol. 2006, 24, 67-78.

39. Hdeib, H. $\omega$-closed mappings. Rev. Colomb. Matem. 1982, 16, 65-78.

40. Al Ghour, S. Certain Covering Properties Related to Paracompactness. Ph.D. Thesis, University of Jordan, Amman, Jordan, 1999.

41. Pareek, C.M. Hereditarily Lindelof and hereditarily almost Lindelof spaces. Math. Japon 1985, 30, 635-639.

42. Al Ghour, S.; Irshedat, B. The topology of $\theta_{\omega}$-open sets. Filomat 2017, 31, 5369-5377.

43. Hussain, S.; Ahmad, B. Soft separation axioms in soft topological spaces. Hacet. J. Math. Stat. 2015, 44, 559-568. [CrossRef] 
44. Solai, R.; Subbiah, V. Soft separation axioms and soft product of soft topological spaces. Maltepe J. Math. 2020, 2, 61-75.

45. Nazmul, S.; Samanta, S.K. Neigbourhood properties of soft topological spaces. Ann. Fuzzy Math. Inform. 2013, 6, 1-15. 